\title{
Replica ai commenti sul caso clinico: Setting ad assetto variabile. La chat dei sogni in psicoterapia: il caso di Adriana
}

\author{
Carmine Parrella*
}

Ringrazio innanzi tutto i colleghi per il contributo offerto dalle loro preziose riflessioni. La realtà di un processo terapeutico è per sua natura multidimensionale, sfaccettata e per molti versi drammaticamente 'incoerente' nel suo fluire caotico di elementi, tanto carichi affettivamente, quanto orfani di una loro collocazione e di un significato dentro una narrazione condivisa. Ogni contributo, nella sua originale prospettiva è una occasione e una possibilità per 'governare' questa complessità.

La terapia stessa è un sognare insieme, nutrito a sua volta dai sogni del terapeuta e del paziente, in un tentativo paradossale di usare i sogni per 'destarsi' da essi.

Le osservazioni dei colleghi mi invitano a riflettere non tanto sulla tecnica o sull'ampliamento della 'cassetta degli attrezzi' per le situazioni di impasse, quanto sul significato transferale e controtransferale di quella scelta.

La possibilità che la chat dei sogni possa sbloccare qualcosa nella relazione terapeutica sta nelle operazioni interne che produce, non tanto in virtù della sua specificità e quindi della sua replicabilità tecnica, quanto come conseguenza del cambiamento di senso che provoca nel paradigma relazionale tra terapeuta e paziente. In questa prospettiva la chat dei sogni è una operazione a due facce, da un lato contiene una spinta evolutiva, dall'altra può essere utilizzata inconsapevolmente in senso difensivo e regressivo dalla coppia terapeuta paziente bloccando le stesse risorse che intenderebbe attivare.

Sento come molto appropriate le osservazioni che l'allargamento del setting siano state un mio 'disperato' tentativo di sfuggire, non ad un'impasse della terapia, ma a ciò che ha prodotto l'impasse. L'allargamento del setting può essere visto come una reazione, un 'agito' a una angoscia paralizzante

*Psicologo e Psicoterapeuta presso Centro di Salute Mentale ASL Toscana Nord Ovest zona di Lucca e socio SIPRe. E-mail: carmine.parrella@uslnordovest.toscana.it 
'mortifera', pericolosa, rischiosa e intollerabile. La chat dei sogni diventa prima che un espediente tecnico, una risposta a un evento emotivo dentro il terapeuta. Il rischio con cui fare i conti è quello della demotivazione del terapeuta, l'abbandono dell'investimento sulla terapia, il considerare il caso incurabile e non trattabile per arrivare poi a replicare la scena traumatica e cioè quella dell'abbandono. Le osservazioni dei colleghi mi danno una serie di elementi, che non voglio analizzare uno per uno ma cogliere nella loro gestalt complessiva, e che tutti insieme, come in una sorta di supervisione a distanza, mi fanno pensare che Adriana non mi parli della sua parte sofferente ma la 'agisca' nella relazione con me. Adriana non mi parla della sua apocalisse, questo è un racconto/copione che conosce molto bene, semplicemente me la fa rivivere. È come se Adriana mi dicesse, 'ecco adesso che anche tu sei in un deserto insieme a me, adesso che ti ho reso impotente come gli altri hanno fatto con me che fai... che ne farai di me... che ne farai di noi?'. Sarai come il papà di peluche dove io posso prendere un po'di calore per poi essere nuovamente abbandonata alla freddezza della mamma di fil di ferro?' È così che la parte sofferente 'preverbale', 'prenatale' di figlia non voluta o parzialmente voluta solo come 'merce di scambio' tra i genitori si materializza. Ed è la difficoltà a riorganizzare il mio spazio psichico per contenere questa angoscia e questa richiesta a cui è impossibile rispondere e rispetto alla quale qualsiasi risposta diretta sarebbe inefficace a spingermi nella direzione di una risposta 'scissa', di proporre cioè uno spazio altro dalla seduta dove poter mettere, non i contenuti che non emergono dal setting (dal setting, infatti, sembra emergere proprio tutto) ma dove poter collocare la mia/nostra impotenza. In questo senso la risposta della chat dei sogni appare come una sorta di scissione creativa, una modalità psichica 'pre-integrata' che permette agli elementi scissi provenienti dalla relazione e proiettati in maniera caotica, di essere 'raccolti', 'contenuti', 'parcheggiati' e di essere tollerati come precondizione per poter essere poi riportati nuovamente in seduta. Proprio mentre scrivo queste righe e rivedo i contributi dei colleghi, mi viene in mente che una nuova posizione possibile nei confronti della bambina deprivata che Adriana mi pone, non è né quella consolatoria fusionale del padre, né quella del 'riparatore' della madre e né quella del terapeuta che l'aiuta a mediare tra mondo del desiderio e mondo della realtà quanto quella dell'amico immaginario che le è mancato quando sperimentava un senso di terribile solitudine e di vuoto. Una delle possibili chiavi di lettura del processo e dei fattori di cura può essere la possibilità di costruire una esperienza 'riparatoria' dove Adriana possa fare nello stesso tempo l'esperienza del vuoto e la presenza di qualcuno accanto a lei. Lei desidera essere vista mentre affronta il vuoto. Il non essere vista l'ha spinta verso soluzioni compensative di seduzione di oggetti onnipotenti da inserire nel suo vuoto. Ciò ha bloccato/condizionato l'attivazione delle sue risorse simboliche interne. Perché, l'esperienza terapeutica 
riparativa si materializzi e sia 'psichicamente' reale e autentica, il terapeuta stesso deve fare esperienza di quella condizione di vuoto senza esserne annientato per poter introdurre nella terapia elementi utili a 'sognare' insieme qualcosa di nuovo e di vitale.

Conflitto di interessi: l'autore dichiara che non vi sono potenziali conflitti di interessi.

Approvazione etica e consenso a partecipare: non necessario.

Ricevuto per la pubblicazione: 5 novembre 2021 .

Accettato per la pubblicazione: 8 novembre 2021 .

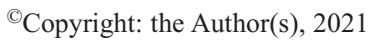

Licensee PAGEPress, Italy

Ricerca Psicoanalitica 2021; XXXII:600

doi:10.4081/rp.2021.600

This article is distributed under the terms of the Creative Commons Attribution Noncommercial License (by-nc 4.0) which permits any noncommercial use, distribution, and reproduction in any medium, provided the original author(s) and source are credited. 
